\title{
On the Stability of the Upside-Down Pendulum with Damping
}

\author{
M. V. Bartuccelli*, G. Gentile ${ }^{\dagger}$, K. V. Georgiou* \\ * Department of Mathematics and Statistics, University of Surrey, Guildford, GU2 7XH \\ $\dagger$ Dipartimento di Mathematica, Università di Roma Tre, Roma, I-00146, Italy
}

July 8, 2009

\begin{abstract}
A rigorous analysis is presented in order to show that, in presence of friction, the upward equilibrium position of the vertically driven pendulum, with a small non-vanishing damping term, becomes asymptotically stable when the period of the forcing is below an appropriate threshold value. As a byproduct we obtain an analytic expression of the solution for initial data close enough to the equilibrium position.
\end{abstract}

Keywords: Mathieu's equation; perturbation theory; stability; basins of attraction

\section{Introduction}

Consider a pendulum of mass $m$ and length $\ell$, constrained to move on a vertical plane under the influence of gravity of intensity $g$ and in presence of friction, with the point of support $P$ subjected to a vertical cosinusoidal oscillation of frequency $\omega$ and amplitude $b$, i.e. $y_{P}=b \cos \omega t$ : the equation of motion for the system (see for instance Landau \& Lifschitz 1976) is given by

$$
\ddot{\theta}+\sin \theta\left(\frac{g}{\ell}-\frac{b \omega^{2}}{\ell} \cos \omega t\right)+\gamma \dot{\theta}=0,
$$

where $\theta \in \mathbb{R} / 2 \pi \mathbb{Z}$ is the angle that the pendulum forms with the downward oriented vertical, $\gamma$ is the dissipation (or friction) coefficient and the dots denote differentiation with respect to $t$.

It is well known (dating back to Stephenson 1908) that the equilibrium state $(\theta, \dot{\theta})=(\pi, 0)$ can be made stable if the frequency and the amplitude of the oscillation are suitably chosen: if $b$ is small (with respect to $\ell$ ), then $\omega$ has to be large enough, say larger than a threshold value $\omega_{0}$. The existing proofs of stability in literature are in absense of friction $(\gamma=0$ in (1.1)) either for the linearized system, which reduces to Mathieu's equation, or based on the averaging (or effective potential) method; one can see Phelps \& Hunter 1965 for the former, and Kapitsa 1951 for the latter (for a review on the averaging method see Arnol'd et al. 1988, Ch. 3).

We refer to Blackburn et al. 1992 for a critical description of the two methods, in particular to understand why they are not completely adequate to solve the problem.

Here we confine ourselves to stress that the averaging method is not rigorous (and even incorrecty in some cases). In fact one introduces an approximation by discarding some terms from the Hamiltonian describing ths system and there is no control on the relevance of the discarded terms. When studying the stability of the inverted state of the pendulum, one obtains for $\omega_{0}$ an approximated value which is in very good agreement with experimental obervations: the problem is that there is no way to conclude theoretically (i.e. without relying a posteriori on the experiments) to what extent the result is reliable, and how relevant can be the error caused by the approximation involved by the method. Even more, when applied to the study of the noninverted state, the averaging method would predict stability for all values of the parameters, in sharp contrast with the fact that such a 
state can be indeed destabilized for some values of the parameters: so for such values the averaging method gives the wrong result.

On the other hand the results found by using the theory of Mathieu's equation apply only to the linearized system, and they cannot be extended immediately to the full nonlinear system (it is well known that linear stability in general gives no information about the stability of the full system).

As a matter of fact, from a rigorous point of view the stability of the upward equilibrium state is far from being obvious: in absense of friction $(\gamma=0$ in (1.1)) the proof requires KAM theory arguments (see for instance Bartuccelli et al. 2000b).

In presence of friction the equilibrium state $(\theta, \dot{\theta})=(\pi, 0)$ is asymptotically stable for the linearized system (for suitable values of the parameters, see below), so that it is expected to remain asymptotically stable when also the nonlinear terms are taken into account. In the present note we give an analytical proof of such a property, by using a technique which has been introduced recently in Classical Mechanics by Eliasson (1988), then developed in various subsequent papers and applied successfully in order to solve several KAM-type problems (for a review we refer to Gentile 1999). Note however, that, with respect to such problems, in our case the analysis is much easier, as there are no small divisors.

Besides stability (which likely would follows - at least for qualitative purposes - from the study of the linearized damped system), our proof gives also an analytical perturbative expression for the solution. More precisely we find that the the solution of the full system is sort of conjugated to the solution of the linear system, in the following sense: by writing (as it is possible, see below) the solution $\theta_{0}(t)$ of the linearized system as

$$
\theta_{0}(t)=e^{-\lambda t} P(\nu t), \quad \nu=\left(\mu_{0}, 2\right), \quad \mu \in(0,1),
$$

with $P$ a $2 \pi$-periodic function in its arguments, then the solution $\theta(t)$ of the full system can be written as

$$
\theta(t)=\Theta\left(e^{-\lambda t}, \nu t\right),
$$

with $\Theta$ a function analytic in its arguments and $2 \pi$-periodic in $\psi \equiv \nu t=\left(\mu_{0} t, 2 t\right)$. The function $\Theta$ can be constructed to all perturbative orders and then shown to be convergent (for initial data close enough to the upward equilibrium state), so that analyticity follows.

Of course the results we find are not so surprising: physically they are exactly the ones expected to hold. Note however that, while on one hand there is a large number of numerical results on the vertically driven pendulum (for a review we refer to Bartuccelli et al. 2000a and to the references quoted therein), on the other hand the analytical results existing on the subject are very few at the moment; in particular the two methods mentioned above, even if they are interesting and often very useful for practical purposes, give only a partial theoretical understanding of the problem, and they are not completely satisfactory from a rigourous point of view, as we noted above.

Moreover we think that our method is interesting by itself: to determine the motion by looking for a solution of some prefixed form is very natural from a physical point of view, and allows to obtain good analytical bounds. The estimates of the basin of attraction can appear to be very poor with respect to those one could obtain numerically, but they turn out to be very appealing if compared with the results provided by the other two analytical methods, with which is not possible to obtain any estimates on the basins of attraction of the equilibrium points.

\section{An analytical proof of asymptotic stability}

With respect to Bartuccelli et al. 2000a, we slightly change the notations, in order to make more terse the comparison with the existing mathematical literature on the subject, especially with Arscott 1964 and Blanch 1965.

If we are interested in the stability of the position $\theta=\pi$ (upside-down pendulum), define $\xi=\pi+\theta$, so that (1.1) becomes

$$
\ddot{\xi}+\sin \xi\left(-\frac{g}{\ell}+\frac{b \omega^{2}}{\ell} \cos \omega t\right)+\gamma \dot{\xi}=0
$$


Define $\omega t=2 \tau$ and $x(\tau)=\xi(t)$; then $(2.1)$ writes

$$
\ddot{x}+\sin x(a+2 q \cos 2 \tau)+2 \lambda \dot{x}=0, \quad a=-\frac{4 g}{\ell \omega^{2}}, \quad q=\frac{2 b}{\ell}, \quad \lambda=\frac{\gamma}{\omega},
$$

(with $\dot{x}=\mathrm{d} x / \mathrm{d} \tau$ and $\ddot{x}=\mathrm{d}^{2} x / \mathrm{d} \tau^{2}$ ), which can be rewritten as

$$
\ddot{x}+f(\tau) x+2 \lambda \dot{x}=[x-\sin x] f(\tau), \quad f(\tau)=a+2 q \cos 2 \tau .
$$

Set

$$
x(\tau)=\delta e^{-\lambda \tau} y(\tau):
$$

then, by writing (2.3) with $\sin x$ expressed as Taylor series of $x$ and dividing it by $\delta e^{-\lambda \tau}$, we obtain

$$
\ddot{y}+f_{0}(\tau) y=f(\tau) \sum_{m=1}^{\infty} \frac{(-1)^{m+1}}{(2 m+1) !} e^{-2 m \lambda \tau} \varepsilon^{m} y^{2 m+1}, \quad \varepsilon=\delta^{2},
$$

where

$$
f_{0}(\tau)=f(\tau)-\lambda^{2}=a_{0}+2 q \cos 2 \tau, \quad a_{0}=a-\lambda^{2} .
$$

In the linear case $(\varepsilon=0)$ the substitution $(2.4)$ is the standard procedure to show that the solutions of Mathieu's equation with damping are conjugated to the solutions of Mathieu's equation; see Jordan \& Smith 1977.

For $\varepsilon=0,(2.5)$ becomes (the standard form of) Mathieu's equation,

$$
\ddot{y}+f_{0}(\tau) y=0, \quad f_{0}(\tau)=a_{0}+2 q \cos 2 \tau ;
$$

we refer to Jordan \& Smith 1977, Ch. 8, for an introductory review and to Arscott 1964 for a more detailed exposition of the subject.

Note that in (2.6) one has $a_{0}<0$ (see $(2.2)$ and $\left.(2.6)\right)$. Let $a_{0}(q), b_{1}(q)$ be the curves of the first characteristic values of Mathieu's equation (see Arscott 1964, Ch. III); for $a_{0}, q$ such that $a_{0}(q)<a_{0}<\min \left\{b_{1}(q), 0\right\}$ the solution of 2.7 is stable, (see Arscott 1964, Ch. VI). More precisely there exist two independent (Floquet) solutions

$$
u_{1}(\tau)=e^{i \mu_{0} \tau} p_{0}(\tau), \quad u_{2}(\tau)=e^{-i \mu_{0} \tau} \bar{p}_{0}(\tau)
$$

where $0<\mu_{0}<1$ (see Arscott 1964 and Blanch 1965), and $p_{0}(\tau)$ is an analytic function of period $\pi$,

$$
p_{0}(\tau)=\sum_{\nu \in \mathbb{Z}} e^{2 i \nu \tau} p_{0 \nu}, \quad p_{0}(0)=1, \quad p_{0}(-\tau)=\bar{p}_{0}(\tau)
$$

here and henceforth, given $z \in \mathbb{C}, \bar{z}$ denotes the complex conjugate of $z$. Both $\mu_{0}$ and $p_{0}(\tau)$ depend on $a_{0}$ and $q$. Note that

$$
\left|p_{0 \nu}\right| \leq P e^{-\kappa|\nu|}, \quad P=\max _{\tau \in[0, \pi]}\left|p_{0}(\tau)\right|
$$

for some $\kappa \in \mathbb{R}_{+}$.

One can construct two real independent solutions as

$$
w_{1}(\tau)=\frac{1}{2}\left[e^{i \mu_{0} \tau} p_{0}(\tau)+e^{-i \mu_{0} \tau} \bar{p}_{0}(\tau)\right], \quad w_{2}(\tau)=\frac{1}{2 i c}\left[e^{i \mu_{0} \tau} p_{0}(\tau)-e^{-i \mu_{0} \tau} \bar{p}_{0}(\tau)\right],
$$

where $c \in \mathbb{R}$ is such that $\dot{w}_{2}(0)=1$. Note that $w_{1}$ is even and $w_{2}$ is odd.

The solution $y^{(0)}(\tau)$ of $(2.7)$ with initial data $\left(y^{(0)}(0), \dot{y}^{(0)}(0)\right)=(A, B) \in \mathbb{R}^{2}$ can be written as

$$
y^{(0)}(\tau)=w_{1}(\tau) A+w_{2}(\tau) B, \quad A^{2}+B^{2}=1,
$$

hence it is in general quasi-periodic (periodic if $\mu_{0}$ is rational), with rotation vector $\omega_{0}=\left(\mu_{0}, 2\right)$. The normalization condition in (2.12) is imposed just for convenience; see (2.16) below.

In terms of the Floquet solutions, (2.12) becomes

$$
y^{(0)}(\tau)=u_{1}(\tau) C+u_{2}(\tau) D=e^{i \mu_{0} \tau} p_{0}(\tau) C+e^{-i \mu_{0} \tau} \bar{p}_{0}(\tau) D
$$


where

$$
C=\frac{A-i B / c}{2}, \quad D=\bar{C}=\frac{A+i B / c}{2} .
$$

Expressed in the variable $x$, the solution $y^{(0)}$ becomes

$$
x^{(0)}(\tau) \equiv \delta e^{-\lambda \tau} y^{(0)}(\tau)=\delta\left[e^{i \mu_{0} \tau-\lambda \tau} p_{0}(\tau) C+e^{-i \mu_{0} \tau-\lambda \tau} \bar{p}_{0}(\tau) D\right],
$$

so that one sees that its limit for $\tau \rightarrow \infty$ tends to 0 : if one neglects the nonlinear terms in $(2.3)$ the origin is asymptotically stable. Note that, in terms of the variable $x$, the initial condition in $(2.12)$ gives

$$
x^{(0) 2}(0)+\dot{x}^{(0) 2}(0)=\delta^{2},
$$

so that $\delta$ measures the distance of the initial datum from the equilibrium point $(0,0)$.

The solution $x^{(0)}$ given by $(2.15)$ can be written as

$$
x^{(0)}(\tau)=\sum_{\nu \in \mathbb{Z}^{2}} e^{i \omega_{0} \cdot \nu \tau-\lambda \tau} x_{\nu}^{(0)}
$$

where $\omega_{0}=\left(\mu_{0}, 2\right), \nu=\left(\nu_{1}, \nu_{2}\right)$, with $\nu_{1}= \pm 1$ and $x_{\left(1, \nu_{2}\right)}^{(0)}=\bar{x}_{-\left(1, \nu_{2}\right)}^{(0)}=\delta p_{0 \nu_{2}} C$; here and henceforth the $\cdot$ denotes the inner product in $\mathbb{Z}^{2}$, so that $\omega_{0} \cdot \nu=\mu_{0} \nu_{1}+2 \nu_{2}$. Hence $x^{(0)}(\tau)$ depends on $\tau$ only through $e^{ \pm i \mu_{0} \tau}, e^{ \pm i 2 \tau}$ and $e^{-\lambda \tau}$. The first two factors give an oscillatory behaviour, while the third one is responsible for the decay towards the origin.

If $\varepsilon \neq 0$ we look for solutions analytic on $\varepsilon$ for $\varepsilon$ small enough, whose dependence on $\tau$ is still through the factors $e^{ \pm i \mu_{0} \tau}, e^{ \pm i 2 \tau}$ and $e^{-\lambda \tau}$. This means that we look for solutions of the form

$$
y(\tau) \equiv y(\tau ; \varepsilon)=\sum_{n=1}^{\infty} \varepsilon^{n} y^{(n)}(\tau)=\sum_{n=1}^{\infty} \varepsilon^{n} \sum_{\nu \in \mathbb{Z}^{2}} \sum_{k=0}^{\infty} e^{i \omega_{0} \cdot \nu \tau-k \lambda \tau} y_{\nu, k}^{(n)}
$$

We shall show that, fixed an arbitrary direction $(A, B)$, with $A^{2}+B^{2}=1$, in the plane $(y, \dot{y})$, the solution $y(\tau)$ of $(2.5)$, with initial condition $(y(0), \dot{y}(0))=\left(y^{(0)}(0), \dot{y}^{(0)}(0)\right)=(A, B)$, remains bounded around the equilibrium point $(0,0)$, provided $\varepsilon$ is small enough. Note that the component $k=0$ already appears when $n=0$, i.e. for $y^{(0)}(\tau)$ : anyway the presence of oscillating (or even constants, that is with $k=0$ and $\nu=0$ ) terms is not a problem because the solution $x(\tau)$ of the equation $(2.3)$ is related to $y(\tau)$ through the change of variable $(2.4)$, and $\theta(t)$ is such that $x(\omega t / 2)=\pi+\theta(t)$.

Then $(x(0), \dot{x}(0))=(\delta A, \delta B)$ is the initial datum for the equation $(2.3)$, hence $(\theta(0), \dot{\theta}(0))=$ $(\pi+\delta A, \delta B)$ is the initial datum for the equation (1.1). By the above discussion we have that any initial datum close enough to the equilibrium point $(\pi, 0)$ generates a solution of $(1.1)$ which is exponentially asymptotic to $(\pi, 0)$ with decay rate $\lambda$ : this implies the asymptotic stability of the position $(\theta, \dot{\theta})=(\pi, 0)$ for the upside-down pendulum.

More formally the result can be stated as follows.

THEOREM. Given the equation (1.1), assume that (for fixed $g$ and $\ell$ ) the parameters $\omega$ and $b$ are such that

$$
a_{0}(2 b / \ell)<-\frac{4 g}{\ell \omega^{2}}-\lambda^{2}<\min \left\{b_{1}(2 b / \ell), 0\right\},
$$

where $q \rightarrow a_{0}(q)$ and $q \rightarrow b_{1}(q)$ are the curves of the first characteristic values of Mathieu's equation. Then the series (2.18) is absolutely convergent for $\varepsilon$ small enough. This means that there exists $\delta_{0}>0$ such that the equilibrium position $(\theta, \dot{\theta})=(\pi, 0)$ is the $\omega$-limit set of any initial datum $(\theta(0), \dot{\theta}(0))$ satisfying $[\theta(0)-\pi]^{2}+\dot{\theta}^{2}(0)<\delta_{0}^{2}$, and the time decay of the solution towards $(\pi, 0)$ is exponential with rate $\lambda$; in particular the ball of center $(\pi, 0)$ and radius $\delta_{0}$ is contained inside the basin of attraction of $(\pi, 0)$. One has $\delta_{0}=O(\sqrt{\lambda})$.

The proof of the above Theorem is carried out in Sect. 3. The condition (2.19) could be easily relaxed into a weaker one. In fact $(2.18)$ requires the solution $y^{(0)}$ to be quasi-periodic: in terms of the parameters $\omega, b$ this means that they lay inside the lowest stability region, so that $\mu_{0}$ is real (see Arscott 1964). Anyway the solution $x^{(0)}$ has a damping factor $e^{-\lambda \tau}$ (see $(2.15)$ ), so that $\mu_{0}$ could be allowed to have also an imaginary part (smaller than $\lambda$ ). As we are not looking for optimal bounds, 
we have neglected such a feature in stating the Theorem; however recall that the damping term destabilizes the motion of the inverted pendulum with respect to the undamped case (see Leiber \& Risken 1988).

Using that $a_{0}(q) \approx-q^{2} / 2$ for $q$ small enough (see Arscott 1964), for $\lambda=0$ the condition (2.19), if $b / \ell \leq 0.450$ so that $\min \left\{b_{1}(2 b / \ell), 0\right\}=0$, reads as $2 g \ell<b^{2} \omega^{2}$, which is the condition found by the averaging method (see Percival \& Richard, Ch. 9, §9.5; compare also Acheson 1993, formula (2.1), for a single pendulum).

\section{Proof of the Theorem}

We look for solutions of the form

$$
y(\tau) \equiv y(\tau ; \varepsilon)=\sum_{n=1}^{\infty} \varepsilon^{n} y^{(n)}(\tau) .
$$

Inserting (3.1) into (2.5), we obtain, by equating the terms of the same order in $\varepsilon$,

$$
\begin{aligned}
& \ddot{y}^{(0)}+f_{0}(\tau) y^{(0)}=0 \\
& \ddot{y}^{(1)}+f_{0}(\tau) y^{(1)}=\frac{1}{3 !} e^{-2 \lambda \tau} f(\tau) y^{(0) 3}, \\
& \ddot{y}^{(2)}+f_{0}(\tau) y^{(2)}=\frac{1}{2} e^{-2 \lambda \tau} f(\tau) y^{(0) 2} y^{(1)}-\frac{1}{5 !} e^{-4 \lambda \tau} f(\tau) y^{(0) 5},
\end{aligned}
$$

and so on; in general, to order $n$, one has

$$
\ddot{y}^{(n)}+f_{0}(\tau) y^{(n)}=F^{(n)}(\tau),
$$

where

$$
\begin{gathered}
F^{(n)}(\tau)=f(\tau) \sum_{m=1}^{n} \frac{(-1)^{m+1}}{(2 m+1) !} e^{-2 m \lambda \tau} \sum_{m^{\prime}=0}^{2 m+1}\left(\begin{array}{c}
2 m+1 \\
m^{\prime}
\end{array}\right) y^{(0) 2 m+1-m^{\prime}}(\tau) \\
\sum_{\substack{n_{1} \geq 1, \ldots, n_{m^{\prime} \geq 1} \geq 1 \\
n_{1}+\ldots+n_{m^{\prime}}=n-m}} \prod_{j=1}^{m^{\prime}} y^{\left(n_{j}\right)}(\tau)
\end{gathered}
$$

The term corresponding to $m^{\prime}=1$ yields $m=n$ and it has to be interpreted as

$$
f(\tau) \frac{(-1)^{n+1}}{(2 n+1) !} e^{-2 n \lambda \tau} y^{(0) 2 n+1}(\tau) ;
$$

note that for $n=1$ it is the only present (see (3.2).

Then the equations (3.3) can be iteratively integrated as

$$
\left(\begin{array}{c}
y^{(n)}(\tau) \\
\dot{y}^{(n)}(\tau)
\end{array}\right)=W(\tau)\left[\left(\begin{array}{c}
y^{(n)}(0) \\
\dot{y}^{(n)}(0)
\end{array}\right)+\int_{0}^{\tau} \mathrm{d} \tau^{\prime} W^{-1}\left(\tau^{\prime}\right)\left(\begin{array}{c}
0 \\
F^{(n)}\left(\tau^{\prime}\right)
\end{array}\right)\right],
$$

where

$$
W(\tau)=\left(\begin{array}{ll}
w_{1}(\tau) & w_{2}(\tau) \\
\dot{w}_{1}(\tau) & \dot{w}_{2}(\tau)
\end{array}\right)
$$

is the Wronskian matrix of the linear system $(2.7)$, so that $W(0)=\mathrm{id}$ and $\operatorname{det} W(\tau)=1$.

Introduce the space $\mathcal{M}$ of functions $h: \mathbb{R} \rightarrow \mathbb{C}$, which admit a formal expansion of the form

$$
h(\tau)=\sum_{\nu \in \mathbb{Z}^{2}} \sum_{k=0}^{\infty} e^{i \nu \cdot \omega_{0} \tau} e^{-k \lambda \tau} h_{\nu, k}
$$


and define

$$
\mathcal{M}_{0}=\left\{h \in \mathcal{M}: h_{0,0}=0\right\} .
$$

Note that

$$
\int_{0}^{\tau} \mathrm{d} \tau^{\prime}\left[e^{i \omega_{0} \cdot \nu \tau^{\prime}-k \lambda \tau^{\prime}}\right]=\frac{e^{i \omega_{0} \cdot \nu \tau-k \lambda \tau}-1}{i \omega_{0} \cdot \nu-k \lambda}
$$

for all $(\nu, k) \in \mathbb{Z}^{2} \times \mathbb{N}$. that

Given a function $h \in \mathcal{M}_{0}$ denote by $\mathcal{I}(h)$ the primitive of $h$ in $\mathcal{M}_{0}$ : then, by (3.10), one can see

$$
\mathcal{I}\left[e^{i \omega_{0} \cdot \nu \tau^{\prime}-k \lambda \tau^{\prime}}\right]=\frac{e^{i \omega_{0} \cdot \nu \tau-k \lambda \tau}}{i \omega_{0} \cdot \nu-k \lambda},
$$

as $h \in \mathcal{M}_{0}$ requires $h_{\nu, k}$ to have $(\nu, k) \neq(0,0)$. The space $\mathcal{M}$ admits a natural decomposition into

$$
\mathcal{M}=\bigoplus_{\nu \in Q} \bigoplus_{k=1}^{\infty} \mathcal{M}_{\nu, k}, \quad Q=\left\{\nu \in \mathbb{Z}^{2}: \nu \sim \nu^{\prime} \text { if } \omega_{0} \cdot \nu=\omega_{0} \cdot \nu^{\prime}\right\}
$$

and $\mathcal{M}_{\nu, k}$ is the space of functions of the form $h(\tau)=a e^{i \omega_{0} \cdot \nu^{\prime} \tau} e^{-k \lambda \tau}$, with $a \in \mathbb{C}$ and for all $\nu^{\prime}$ in the same equivalence class of $\nu$. Note that $Q=\mathbb{Z}^{2}$ if the components of $\omega_{0}$ are rationally independent. By construction $\mathcal{I}: \mathcal{M}_{\nu, k} \rightarrow \mathcal{M}_{\nu, k}$.

Define the function $w_{12}$ as

$$
w_{12}\left(\tau, \tau^{\prime}\right)=\left[w_{2}(\tau) w_{1}\left(\tau^{\prime}\right)-w_{1}(\tau) w_{2}\left(\tau^{\prime}\right)\right]=\frac{1}{2 i c}\left[u_{1}(\tau) u_{2}\left(\tau^{\prime}\right)-u_{2}(\tau) u_{1}\left(\tau^{\prime}\right)\right]
$$

One can write explicitly (3.6) as

$$
\begin{aligned}
\left(\begin{array}{l}
y^{(n)}(\tau) \\
\dot{y}^{(n)}(\tau)
\end{array}\right)= & \left(\begin{array}{l}
w_{1} y^{(n)}(0)+w_{2} \dot{y}^{(n)}(0) \\
\dot{w}_{1} y^{(n)}(0)+\dot{w}_{2} \dot{y}^{(n)}(0)
\end{array}\right) \\
& +\int_{0}^{\tau} \mathrm{d} \tau^{\prime}\left(\begin{array}{l}
{\left[w_{2}(\tau) w_{1}\left(\tau^{\prime}\right)-w_{1}(\tau) w_{2}\left(\tau^{\prime}\right)\right] F^{(n)}\left(\tau^{\prime}\right)} \\
{\left[\dot{w}_{2}(\tau) w_{1}\left(\tau^{\prime}\right)-\dot{w}_{1}(\tau) w_{2}\left(\tau^{\prime}\right)\right] F^{(n)}\left(\tau^{\prime}\right)}
\end{array}\right) .
\end{aligned}
$$

Choose (for simplicity) initial data such that $\left(y^{(n)}(0), \dot{y}^{(n)}(0)\right)=(0,0)$ : this means that the initial datum remains $(y(0 ; \varepsilon), \dot{y}(0 ; \varepsilon))=\left(y^{(0)}(0), \dot{y}^{(0)}(0)\right)=(A, B)$ for all $\varepsilon$ for which the solution $y(\tau ; \varepsilon)$ is defined.

Then (3.14) gives for the first component

$$
y^{(n)}(\tau)=\int_{0}^{\tau} \mathrm{d} \tau^{\prime} w_{12}\left(\tau, \tau^{\prime}\right) F^{(n)}\left(\tau^{\prime}\right)==\mathcal{I}\left(w_{12} F^{(n)}\right)(\tau)-\mathcal{I}\left(w_{12} F^{(n)}\right)(0) .
$$

In particular one has

$$
y^{(1)}(\tau)=\sum_{\sigma=0,1} \mathcal{I}\left(\frac{1}{3 !} w_{12}\left(\tau, \tau^{\prime}\right) e^{-2 \lambda \tau^{\prime}} f\left(\tau^{\prime}\right) y^{(0) 3}\left(\tau^{\prime}\right)\right)(\sigma \tau)
$$

for the first order term; see (3.2).

By expressing $y^{(n)}$ as in (3.15) and developing $F^{(n)}$ as in (3.4), one obtains an (integral) expression of $y^{(n)}$ in terms of functions $y^{\left(n^{\prime}\right)}$, with $n^{\prime}<n$. One can iterate the procedure until only terms $y^{(1)}$ given by (3.16) appear. In such a way one is naturally led to represent $y^{(n)}$ in terms of tree graphs (or simply trees). We refer to Harary \& Palmer 1973 for an introduction to the theory of graphs; our construction follows very closely Gallavotti 1994.

A tree $\theta$ is a partially ordered set of points and lines connecting the points. The partial ordering is from right to left and it will be denoted by $\preceq$. The leftmost point $r$ is called the root of the tree, while all the other points are called nodes and are denoted by labels $v$; by the definition of the order relation one has $r \succ v$ for all vertices $v$. The lines are denoted by $\ell$; they are all oriented towards the root. If a line $\ell$ connects a node $v_{2}$ to a node $v_{1} \succ v_{2}$, we shall write $\ell=\ell_{v_{2}}$ and $v_{2}^{\prime}=v_{1}$ : we say that the line enters $v_{1}$ and exits $v_{2}$, and that $v_{1}$ is the node immediately following $v_{2}$. The line $\ell_{0}$ entering the root is called the root line: if we denote by $v_{0}$ the last node of the tree, then $\ell_{0}=\ell_{v_{0}}$. The first nodes of a tree, i.e. the nodes which do not follow any other nodes, are called the endpoints 


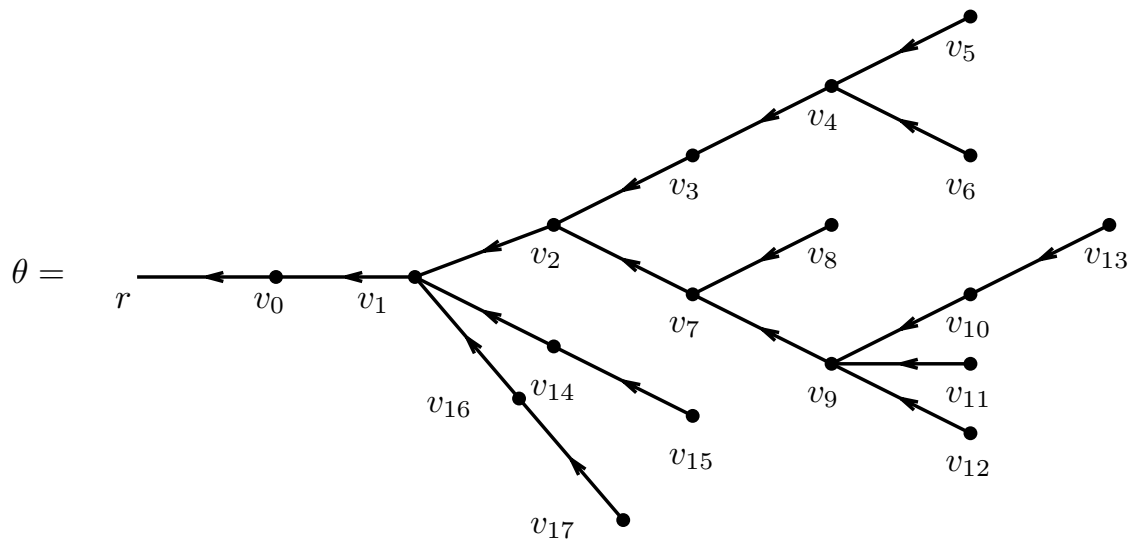

Figure 1: A tree $\theta$ with 18 nodes and 8 endpoints.

of the tree. An example of tree is given in Figure 1. We shall call $V(\theta)$ the set of nodes in $\theta$ and $\Lambda(\theta)$ the set of lines in $\theta$ : by construction $|V(\theta)|=|\Lambda(\theta)|$.

Given a tree $\theta$ and a node $v \in V(\theta)$, the set of nodes $w \preceq v$ and of lines connecting them is still a tree graph: we call it a subtree of $\theta$. Its root is the node $v^{\prime}$ of $\theta$, and its root line is the line $\ell_{v}$, so that we can denote the subtree by $\theta_{v}$, by explicitly assigning its last node (so uniquely identifying the root), and say that $\theta_{v}$ enters $v^{\prime}$. By construction $\theta=\theta_{v_{0}}$.

If we write $y^{(0)}$ as in (2.12) we can expand in (3.4)

$$
y^{(0) 2 m+1-m^{\prime}}(\tau)=\sum_{m_{1}=1}^{2 m+1-m^{\prime}}\left(\begin{array}{c}
2 m+1-m^{\prime} \\
m_{1}
\end{array}\right)\left(w_{1}(\tau) A\right)^{m_{1}}\left(w_{2}(\tau) B\right)^{2 m+1-m^{\prime}-m_{1}} .
$$

To each node we associate the labels $m_{v}, m_{v 1}, m_{v 2}$ and $m_{v 3}$, which are positive integers satisfying the constraint that

$$
m_{v 1}+m_{v 2}+m_{v 3}=2 m_{v}+1 .
$$

The label $m_{v}$ is called the order label, while $m_{v 3}$ is the branching label: the latter denotes the number of lines entering the node $v$; for instance in Figure 1 one has $m_{v_{1} 3}=3, m_{v_{2} 3}=2, m_{v_{3} 3}=1$, $m_{v_{4} 3}=2, m_{v_{5} 3}=0$ and so on.

We define the order $n$ of a tree as

$$
n=\sum_{v \in V(\theta)} m_{v}
$$

To each node $v$ we associate also a time variable $\tau_{v}$, a function

$$
\mathcal{A}_{v}\left(\tau_{v}\right) \equiv \frac{1}{m_{v 1} ! m_{v 2} ! m_{v 3} !}(-1)^{m_{v}+1} e^{-2 m_{v} \lambda \tau_{v}}\left(w_{1}\left(\tau_{v}\right) A\right)^{m_{v 1}}\left(w_{2}\left(\tau_{v}\right) B\right)^{m_{v 2}},
$$

a function $w_{12}\left(\tau_{v^{\prime}}, \tau_{v}\right)$, and a label $\sigma_{v} \in\{0,1\}$. To the root $r$ we associate a time variable $\tau_{r} \equiv t$.

Note that one can associate the functions $w_{1}\left(\tau_{v^{\prime}}\right)$ and $w_{2}\left(\tau_{v^{\prime}}\right)$ in $w_{12}\left(\tau_{v^{\prime}}, \tau_{v}\right)$ to the node $v^{\prime}$ : in such a way to each node $v$ we can associate a label $\delta_{v} \in\{1,2\}$ and a label $\rho_{v} \in\{1,2\}$, with $\rho_{v}+\delta_{v}=3$, and write

$$
w_{12}\left(\tau_{v}^{\prime}, \tau_{v}\right)=\sum_{\delta_{v}+\rho_{v}=3}(-1)^{\delta_{v}+1} w_{\rho_{v}}\left(\tau_{v^{\prime}}\right) w_{\delta_{v}}\left(\tau_{v}\right)
$$

Therefore we can associate to each node $v \in V(\theta)$ a node function

$$
\mathcal{B}_{v}\left(\tau_{v}\right)=(-1)^{\delta_{v}+1} w_{\delta_{v}}\left(\tau_{v}\right) \mathcal{A}_{v}\left(\tau_{v}\right) \prod_{w: w^{\prime}=v} w_{\rho_{w}}\left(\tau_{v}\right)
$$

and to the root a root function

$$
\mathcal{B}_{r}(t)= \begin{cases}w_{1}(t) & \text { if } \delta_{v_{0}}=2 \\ w_{2}(t) & \text { if } \delta_{v_{0}}=1\end{cases}
$$




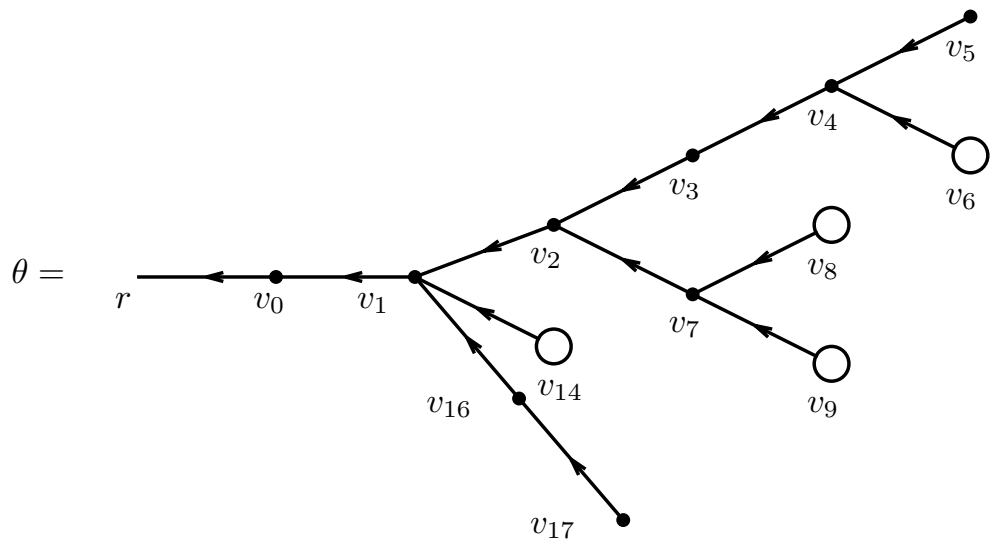

Figure 2: A reduced tree $\bar{\theta}$ with 9 free nodes and 4 leaves. It has been obtained by the tree represented in Figure 1, by encircling inside bubbles all nodes $v_{i}$ with $\sigma_{v_{i}}=0$, then deleting all the bubbles except the outer ones, hence representing the subtrees enclosed by the remaining bubbles as white balls. There are 6 endpoints, two of which $\left(v_{5}\right.$ and $\left.v_{17}\right)$ are free nodes. One has by construction $\sigma_{v_{0}}=1, \sigma_{v_{1}}=1, \sigma_{v_{2}}=1, \sigma_{v_{3}}=1, \sigma_{v_{4}}=1, \sigma_{v_{5}}=1, \sigma_{v_{7}}=1, \sigma_{v_{16}}=1$ and $\sigma_{v_{17}}=1$, while $\sigma_{v_{6}}=0$, $\sigma_{v_{8}}=0, \sigma_{v_{9}}=0$ and $\sigma_{v_{14}}=0$.

Finally to each node $v$ we associate a value $\operatorname{Val}\left(\theta_{v}\right)$, which is iteratively defined as follows. For $v \prec v_{0}$ one sets

$$
\operatorname{Val}\left(\theta_{v}\right)=\mathcal{I}\left(\mathcal{B}_{v}\right)\left(\tau_{v^{\prime}}\right)-\mathcal{I}\left(\mathcal{B}_{v}\right)(0)=\sum_{\sigma_{v}=0,1} \mathcal{I}\left(\mathcal{B}_{v}\right)\left(\sigma_{v} \tau_{v^{\prime}}\right)
$$

if $v$ is an endpoint, and

$$
\begin{aligned}
\operatorname{Val}\left(\theta_{v}\right) & =\mathcal{I}\left(\mathcal{B}_{v} \operatorname{Val}\left(\theta_{1}\right) \ldots \operatorname{Val}\left(\theta_{m_{v 3}}\right)\right)\left(\tau_{v^{\prime}}\right)-\mathcal{I}\left(\mathcal{B}_{v} \operatorname{Val}\left(\theta_{1}\right) \ldots \operatorname{Val}\left(\theta_{m_{v 3}}\right)\right)(0) \\
& =\sum_{\sigma_{v}=0,1} \mathcal{I}\left(\mathcal{B}_{v} \operatorname{Val}\left(\theta_{1}\right) \ldots \operatorname{Val}\left(\theta_{m_{v 3}}\right)\right)\left(\sigma_{v} \tau_{v^{\prime}}\right),
\end{aligned}
$$

if $v$ is not an endpoint, while for $v=v_{0}$ one sets

$$
\operatorname{Val}(\theta)=\sum_{\sigma_{v_{0}}=0,1} \mathcal{B}_{r}(t) \mathcal{I}\left(\mathcal{B}_{v_{0}} \operatorname{Val}\left(\theta_{1}\right) \ldots \operatorname{Val}\left(\theta_{m_{v_{0} 3}}\right)\right)\left(\sigma_{v_{0}} t\right)
$$

Each time $\sigma_{v}=0$ for some $v \in V(\theta)$ the subtree $\theta_{v}$ contributes a constant factor (i.e. independent of time) to the value of the tree $\theta$ : in general we have a product of several factorizing terms. This can be better understood graphically in terms of leaves (as done in Gentile 1995, with respect to which, however, we proceed in a slightly different way). Given a tree $\theta$, let us define the reduced tree $\bar{\theta}$ in the following way. For each node $v \prec v_{0}$ with $\sigma_{v}=0$ let us draw a bubble encircling the node together with the entire subtree $\theta_{v}$, and let us delete all the so obtained bubbles, but the outer ones (i.e. the "maximal bubbles"); each remaining bubble encloses a subtree with last node $v$ and $\sigma_{v}$ label fixed to be zero, and it becomes an endpoint $v$ which can be graphically represented as a white ball attached to the node $v^{\prime}$ through a line $\ell_{v}$; see Figure 2. We call leaf such an endpoint.

We call free nodes the reduced tree nodes different from the leaves; the leaves will be considered a particular type of endpoints, but they will be distinguished from the free nodes. We define the reduced order of a reduced tree as the sum $n_{0}$ of the labels $m_{v}$ associated to its free nodes; by construction $n_{0} \leq n$ (the equality holding only if there are no leaves). We can associate to a reduced tree $\bar{\theta}$ a value $\operatorname{Val}(\bar{\theta})$, where, corresponding to each free node $v$, there is a factor $\mathcal{B}_{v}$ and, corresponding to each leaf $v$, there is factor $L_{v}$, which will be called the value of the leaf: a leaf $v$ contributes to $\operatorname{Val}(\theta)$ only through a factor $w_{\rho_{v}}\left(\tau_{v^{\prime}}\right)$ (which is taken into account in the node function $\mathcal{B}_{v^{\prime}}$, see $(3.22)$ ).

Each leaf value $L_{v}$ can be expressed as a tree value $\operatorname{Val}\left(\theta_{v}\right)$, provided that one sets $\sigma_{v}=0$. The tree value $\operatorname{Val}(\theta)$ will be simply the product of the value of the reduced tree $\operatorname{Val}(\bar{\theta})$ times the values of its leaves: each leaf value $L_{v}$ in turn is given by the product of the value of a reduced tree $\bar{\theta}_{v}$ times the product of the values of its leaves, and so on. Then in the following we can confine ourselves to 
consider the case in which $\sigma_{v}=1$ for all $v \in V(\theta)$, provided the nodes can be allowed to represent also leaves $w$ contributing the functions $w_{\sigma_{w}}\left(\tau_{v}\right)$ to the value of the (reduced) tree.

If we recall the expression (3.22) for the node functions $\mathcal{B}_{v}$, we immediately see that each node function can be decomposed into

$$
\mathcal{B}_{v}\left(\tau_{v}\right)=e^{-2 m_{v} \lambda \tau} \sum_{\nu \in \mathbb{Z}^{2}} e^{i \nu \cdot \omega_{0} \tau_{v}} \mathcal{B}_{v \nu},
$$

with

$$
\mathcal{B}_{v \nu}=e^{2 m_{v} \lambda \tau}\left[(-1)^{\delta_{v}+1} w_{\delta_{v}} \mathcal{A}_{v} \prod_{w: w^{\prime}=v} w_{r_{w}}\right]_{\nu_{v}},
$$

where the exponential $e^{2 m_{v} \lambda \tau}$ simply deletes the corresponding $e^{-2 m_{v} \lambda \tau}$ arising from $\mathcal{A}_{v}$ (see (3.19)).

Then $\nu_{v}=\left(\nu_{v 1}, \nu_{v 2}\right) \in \mathbb{Z}^{2}$ is called the mode label associated to the node $v$ and represents the Fourier label of the function in (3.22).

For the root we set

$$
\mathcal{B}_{r \nu_{r}}= \begin{cases}w_{1 \nu_{r}} & \text { if } \delta_{v_{0}}=2, \\ w_{2 \nu_{r}} & \text { if } \delta_{v_{0}}=1,\end{cases}
$$

and call $\nu_{r} \in \mathbb{Z}^{2}$ the mode label of the root.

Define the momentum flowing through a line $\ell_{v}$ and the decay label, respectively, as

$$
\nu_{\ell_{v}}=\sum_{w \preceq v} \nu_{v}, \quad k_{\ell_{v}}=\sum_{w \preceq v} 2 m_{v} .
$$

In this way we have that the operator $\mathcal{I}$ corresponding to a node $v$ acts as $\mathcal{I}$ applied to the exponential $\exp \left[i \omega_{0} \cdot \nu_{\ell_{v}} \tau_{v}-k_{\ell_{v}} \lambda \tau_{v}\right]$, i.e. as in (3.10), with $\nu \equiv \nu_{\ell_{v}}$ and $k=k_{\ell_{v}}$. Therefore we can represent the value of the tree not by associating to the nodes the $\mathcal{I}$ operators, but using the Fourier expansion (3.27) for the node functions and associating to the any line $\ell$ exiting from a free node a propagator

$$
g_{\ell} \equiv \frac{1}{\left(i \omega_{0} \cdot \nu_{\ell}-k_{\ell} \lambda\right)},
$$

while we simply set $g_{\ell}=1$ if $\ell$ exits from a leaf.

Finally we shall define the node factor and the root factor, respectively, as

$$
\left\{\begin{array}{ll}
F_{v}=\mathcal{B}_{v \nu_{v}}, & \text { if } v \text { is a free node, } \\
F_{v}=1, & \text { if } v \text { is a leaf },
\end{array} \quad F_{r}=\mathcal{B}_{r \nu_{r}} .\right.
$$

By writing $y^{(n)}(\tau)$ as in (2.18), then we can write, by also redefining the value of the tree with respect to the previous definition,

$$
y_{\nu, k}^{(n)}=\sum_{\theta \in \mathcal{T}_{\nu, k}^{n}} \operatorname{Val}(\theta), \quad \operatorname{Val}(\theta)=F_{r}\left[\prod_{v \in V(\theta)} F_{v}\right]\left[\prod_{\ell \in \Lambda(\theta)} g_{\ell}\right],
$$

if $\mathcal{T}_{\nu, k}^{n}$ is the set of all trees of order $n$ such that

$$
\nu_{r}+\nu_{\ell_{0}}=\nu, \quad k_{\ell_{v_{0}}}=k,
$$

if $\nu_{\ell_{0}}$ is the momentum flowing through the root line, $\nu_{r}$ is the mode label of the root and $k_{\ell_{v_{0}}}$ is the decay label associated to the root line. Note that $k=n_{0} \leq n$.

Now it is easy to show that for all trees $\theta \in \mathcal{T}_{\nu, k}^{n}$, one has

$$
\sum_{\left\{\nu_{v}\right\}_{v \in \theta}}\left|F_{r} \prod_{v \in V(\theta)} F_{v}\right| \leq B_{1} C_{1}^{n} e^{-k^{\prime}|\nu|},
$$

for some constants $k^{\prime} \in(0, \kappa)$ and $B_{1}, C_{1}$ positive, while the number of trees in $\mathcal{T}_{\nu, k}^{n}$ with fixed mode labels is bounded by $C_{2}^{k}$ for some constant $C_{2}$; the summability on $\left\{\nu_{v}\right\}$ in (3.35) can be performed 
(neglecting the constraint that a momentum $\nu-\nu_{r}$ has to flow through the root line) by using the property (2.10), in a standard way; see for instance Gentile \& Mastropietro 1996.

Moreover one has

$$
\left|g_{\ell}\right| \leq \frac{1}{\lambda}
$$

as $k_{\ell} \geq 2$ (see $\left.(3.30)\right)$ : in particular no condition has to be imposed on the rotation vector $\omega_{0}$. In terms of the parameters $\omega, b$, we require only that they lay inside the lowest stability region.

By collecting together all the bounds found above, we have that

$$
\left|y^{(n)}\right| \leq B_{0} C_{1}^{n} C_{2}^{n} \lambda^{-n}
$$

for some constant $B_{0}$ so that the series (3.1) defining $y(\tau ; \varepsilon)$ converges for $|\varepsilon|<\varepsilon_{0}=O(\lambda)$.

The discussion in this section leads to the following result stating the asymptotic stability of the position $(y, \dot{y})=(0,0)$. Given the equation (2.5), assume that the parameters $\omega$ and $b$ are such that (2.19) is satisfied; then there exists $\varepsilon_{0}>0$ such that the equilibrium position $(y, \dot{y})=(0,0)$ is the $\omega$-limit set of any initial datum $(y(0), \dot{y}(0))$ satisfying $y^{2}(0)+\dot{y}^{2}(0)<\varepsilon_{0}^{2}$. In particular the ball of center $(0,0)$ and radius $\varepsilon_{0}$ is contained inside the basin of attraction of $(0,0)$. One has $\varepsilon_{0}=O(\lambda)$. By the discussion in Sect. 2 this implies the last assertion of the Theorem (recall also that $\delta=\sqrt{\varepsilon}$, for $\varepsilon \in \mathbb{R}^{+}$). Finally, the convergence of the series (2.18) and the definition of the change of variables (2.4) imply that the solutions $\theta(t)$, with initial conditions inside the basin of attraction of $(\theta, \dot{\theta})=(\pi, 0)$ converge to such point exponentially, at a rate which, in terms of $(2.2)$ reads as $\lambda$.

\section{Comments and conclusions}

The analytical result stated by the Theorem can also be numerically checked. To accomplish this, we have used a Mathematica program which numerically integrates the equation of motion (2.1). As we expect, due to dissipation, the angle the pendulum makes with the upward vertical terminates at $(\xi, \dot{\xi})=(0,0)$, which is the inverted fixed point. The analysis performed in the previous section shows that there exists a quasi-periodic function $P_{0}(t)$ such that

$$
\xi(t)-e^{-\lambda t} P_{0}(t)=O\left(e^{-2 \lambda t}\right), \quad \xi(t)=\pi+\theta(t),
$$

so that the function $e^{\lambda t} \xi(t)$ is quasi-periodic up to corrections $O\left(e^{-\lambda t}\right)$. Therefore the corrections terms tend to disappear after a relatively short time, and a bounded quasi-periodic function is left after some transient: this is illustrated in Figure 3, for values of the parameters $a=0.40, q=1.09$ and $\lambda=0.08$, and for initial conditions $(\xi(0), \dot{\xi}(0))=(0.04,0)$.

The same result was obtained with a $\mathrm{C}$ program; moreover the Fourier analysis (see Figure 4 ) of the function $P_{0}(\nu t)$ shows a power spectrum with two basic frequencies $\nu=\left(\mu_{0}, 2\right)$, with $\mu_{0}=0.4765$, according to (1.3).

Also the numerical computation of the largest Lyapunov exponent for the downward state (with the same C program used in Bartuccelli et al. 2000a), at the fixed values of the parameters, gives the value $\lambda$, in excellent agreement with the results found analytically.

In our analytical estimates no optimality has been looked for. Note that our bounds work for any kind of perturbation with respect to the linear case, (we simply show in Sect. 3 that the nonlinear terms do not worsen the conditions of stability holding in the linear case), so they do not distinguish between an interaction $\cos \theta$ (as the vertically driven pendulum yields) and, for instance, an interaction $\cosh \theta$ : of $\operatorname{course}$ in the first case a larger basin of attraction is expected. Note also that we explicitly use the parity of the perturbation in order to obtain an expansion in powers of $\varepsilon=\delta^{2}$ (see Sect. 3): however this is not at all an essential feature, and the analysis can be easily adapted to any kind of analytic perturbation (simply expanding directly in powers of $\delta$ ). It would be interesting to try to take advantage of the exact form of the interaction in order to improve the bounds: as a matter of fact the nonlinear terms could even improve such conditions, and this is what likely happens physically. For the reason explained above the bounds of the stability regions (in phase space) are far worse than the experimentally observable ones (see Acheson \& Mullin 1993). 


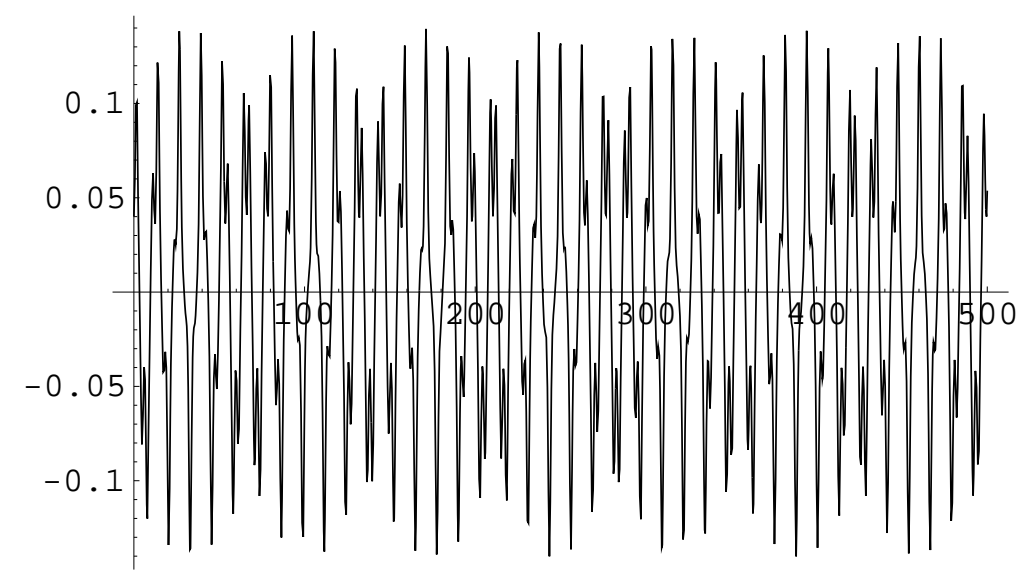

Figure 3: Time history of $e^{\lambda t} \xi(t)$, where $\xi(t)=\pi+\theta(t)$ and $\theta(t)$ is the angle the pendulum makes with the downward vertical and $\lambda$ is given by (2.2). With reference to equation (2.2) one has $a=0.40, q=1.09$ and $\lambda=0.08$; the initial conditions are $(\xi(0), \dot{\xi}(0)=(0.04,0)$.

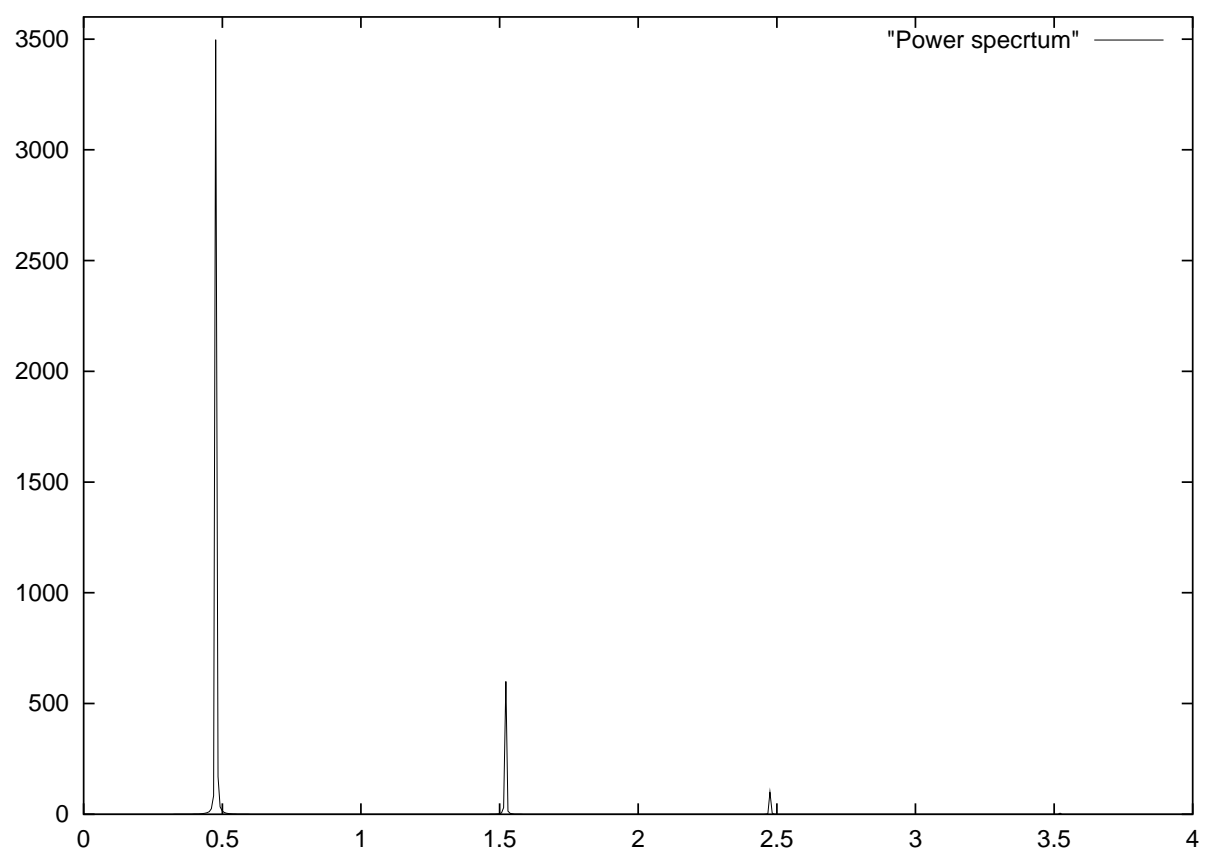

Figure 4: Power spectrum of the function $e^{\lambda t} \xi(t)$. There are (decreasing) peaks at frequencies 0.4765, $1.5235,2.4765,3.5235$ (not detectable in the Figure, but deducible from the numerical data), ..., which reveal the existence of two basic frequencies $\nu=(0.4765,2)$, according to (1.3). 
The bound on the diameter of the basin of attraction given by the Theorem is not uniform in $\lambda$ (it shrinks to zero for $\lambda \rightarrow 0$ ). On the other hand, in absence of friction, the equilibrium position $(\pi, 0)$ is expected to be stable for $\omega$ large enough,

Within the linear approximation, in absense of friction, a bound of the stability region in the plane $(a, q)$ is given in Acheson 1993. If one wants to take into account also the nonlinear terms, one can use KAM type arguments to prove rigorously the stability for the undamped pendulum; see Bartuccelli et al. 2000b.

In absence of friction and within the linear approximation, Acheson (1993) studies also the dynamics of several pendula hanging down, one from another: he finds conditions on the proper frequencies of the system under which the configuration with all the pendula turned upside-down becomes stable. Note however that his results do not carry over to the full nonlinear system, because no stability is in general expected in the case of more than one pendulum. Indeed in such a case the existence of KAM tori (which could be easily proved also in such a case) is no more sufficient to prevent the so-called Arnol'd diffusion: in particular the action variables can drift far away from the initial values (see Arnol'd et al. 1988, Ch. 5, §3.4). However one could prove stability on long times (of Nekhoroshev type), for instance by following the methods in Guzzo et al. 1998.

On the other hand the friction should have a stabilizing effect as in the case of a single pendulum. In the linear approximation the configuration with all the pendula inverted, which is stable in absence of friction (as shown in Acheson 1993), should become asympotically stable, hence attracting, in presence of friction, and the asymptotic stability should persist in the nonlinear regime (as it is shown in the present paper for the case of a single pendulum). This is in agreement with the experimental results by Acheson \& Mullin (1993), for the double and triple pendula, for which the inverted configurations are found to be stable when the pivot is subjected to a rapid vertical oscillation: in fact the pendula tend to wobble towards the upward vertical.

Note that in presence of friction, even for a single pendulum, also attracting periodic orbits can arise in numerical simulations, so that, for initial data not too close to the equilibrium positions, other asympotics could be found.

\section{Acknowledgements}

We thank G. Gallavotti for useful discussions. We are also indebted to F. Bonetto for providing us with his numerical $\mathrm{C}$ programs for computing orbits and power spectra of the solutions of the equations of motion.

\section{References}

D. J. Acheson, A pendulum theorem, Proc. R. Soc. Lond. A 443 (1993), 239-245.

D. J. Acheson \& T. Mullin, Upside-down pendulums, Nature 366 (1993), 215-216.

V. I. Arnol'D, V. V. Kozlov \& A. I. Neishtadt, Dynamical Systems III. Mathematical aspects of classical and celestial mechanics, Springer, Berlin, 1988.

F. M. Arscott, Periodic differential equations, Pergamon Press, Oxford, 1964.

M. V. Bartuccelli, G. Gentile \& K. V. Georgiou, On the dynamics of a vertically driven damped planar pendulum, Preprint (2000a).

M. V. Bartuccelli, G. Gentile \& K. V. Georgiou, KAM theorem and stability of the upsidedown pendulum, Preprint (2000b).

J. A. Blackburn, H. J. T. Smith \& N. Grønbech-Jensen, Stability and Hopf bifurcations in an inverted pendulum, Amer. J. Phys. 60 (1992), 903-908.

G. Blanch, Mathieu functions, in M. Abromowitz \& I. A. Stegun, Handbook of mathematical functions, Dover, New York, 1965.

L. H. Eliasson, Absolutely convergent series expansions for quasi-periodic motions, University of Stockholm preprint (1988), and math. Phys. Electron. J. 2, No. 4 (1996). 
G. Gallavotti, Twist KAM tori, quasi flat homoclinic intersections, and other cancellations in the perturbation series of certain completely integrable hamiltonian systems. A review, Rev. Math. Phys. 6 (1994), 343-411.

G. Gentile, Whiskered tori with prefixed frequencies and Lyapunov spectrum, Dynam. Stability of Systems 10 (1995), 269-308.

G. Gentile, Diagrammatic techniques in perturbation theory, and applications, Proceedings of the Workshop on "Symmetry and Perturbation Theory", Rome (1998), World Scientific, Singapore, 1999.

G. Gentile \& V. Mastropietro, Methods for the analysis of the Lindstedt series for KAM tori and renormalizability in classical mechanics. A review with some applications, Rev. Math. Phys. 8 (1996), 393-444.

M. Guzzo, F. Fassò \& G. Benettin, On the stability of elliptic equilibria, Math. Phys. Electron. J. 4 (1998), Paper 1, 1-16.

F. Harary \& E. PAlmer, Graphical enumerations, Academic Press, New York, 1973.

D. W. Jordan \& P. Smith Nonlinear ordinary differential equations, Clarendon Press, Oxford, 1977.

T. Leiber \& H. Risken, Stability of parametrically exited dissipative systems, Phys. Lett. A 129 (1988), 214-218.

P. L. KAPITSA, Dynamic stability of a pendulum with a vibrating point of suspension, Zh. Ehksp. Teor. Fiz. 21 (1951), 588-598 (Russian), translated version in Collected papers by P. L. Kapitsa, edited by D. ter Haar, Pergamon, Vol. 2, 714-726, London, 1965.

L. D. Landau, E. M. Lifshitz, Mechanics, 3rd Edn, Pergamon Press, Oxford, 1976.

I. Percival \& D. Richards, Introduction to dynamics, Cambridge University Press, Cambridge, 1982.

F. M. Phelps, III \& J. H. Hunter, JR., An analytical solution of the inverted pendulum, Amer. J. Phys. 33 (1965), 285-295.

A. Stephenson, On a new type of dynamical stability, Mem. Proc. Manch. Lit. Phil. Soc. 52 (1908), 1-10. 